\title{
Blume-Emery-Griffiths spin glass and inverted tricritical points
}

\author{
V. Ongun Özçelik ${ }^{1}$ and A. Nihat Berker ${ }^{2,3,4}$ \\ ${ }^{1}$ Department of Physics, Istanbul Technical University, Maslak 34469, Istanbul, Turkey \\ ${ }^{2}$ College of Sciences and Arts, Koç University, Sarlyer 34450, Istanbul, Turkey \\ ${ }^{3}$ Department of Physics, Massachusetts Institute of Technology, Cambridge, Massachusetts 02139, USA \\ ${ }^{4}$ Feza Gürsey Research Institute, TÜBITTAK-Bosphorus University, Çengelköy 34684, Istanbul, Turkey \\ (Received 18 March 2008; revised manuscript received 30 June 2008; published 3 September 2008)
}

\begin{abstract}
The Blume-Emery-Griffiths spin glass is studied by renormalization-group theory in $d=3$. The boundary between the ferromagnetic and paramagnetic phases has first-order and two types of second-order segments. This topology includes an inverted tricritical point, first-order transitions replacing second-order transitions as temperature is lowered. The phase diagrams show disconnected spin-glass regions, spin-glass and paramagnetic reentrances, and complete reentrance, where the spin-glass phase replaces the ferromagnet as temperature is lowered for all chemical potentials.
\end{abstract}

DOI: 10.1103/PhysRevE.78.031104

PACS number(s): 75.10.Nr, 05.50.+q, 61.43.-j, 64.60.aq

The Blume-Emery-Griffiths (BEG) model $[1,2]$ is the simplest system for the study of the various meetings of firstand second-order phase boundaries between ordered and disordered phases, in a plethora of phase diagram topologies [3]. In these diagrams, the second-order phase transitions are dominated by thermal fluctuations and occur at high temperatures. The first-order phase transitions evolve, to finite temperatures, from zero-temperature ground-state energy crossings and occur at low temperatures. In a well-known phase diagram topology, a tricritical point separates the hightemperature second-order boundary and the low-temperature first-order boundary. The BEG model has been used to describe ${ }^{3} \mathrm{He}-{ }^{4} \mathrm{He}$ mixtures [1], solid-liquid-gas systems [4], multicomponent fluid and liquid-crystal mixtures [5], microemulsions [6], semiconductor alloys $[7,8]$, and electronic conduction systems [9].

The inclusion of frozen disorder (quenched randomness) to these systems should yield new phase diagrams, as is indeed seen in our current work. We find that a temperature sequence of transitions that is reverse to the above can occur with the inclusion of quenched randomness. Thus, an inverted tricritical point is obtained, separating a hightemperature first-order boundary and a low-temperature second-order boundary. Since the BEG model is the generic model for tricriticality, we believe that the quenched randomness effect of inverted tricriticality should be quite generally applicable. With frustrated quenched randomness [10-13], the spin-glass phase appears within the Blume-EmeryGriffiths global phase diagram (Fig. 1). Thus, a new spinglass phase diagram topology is found, in which disconnected spin-glass regions occur close to the ferromagnetic and antiferromagnetic phases, but are separated by a paramagnetic gap. No experimental spin-glass phase diagram has to our knowledge as yet yielded disconnected spin-glass regions. However, if the global phase diagram of a physical realization of the BEG spin glass is fully explored, these disconnected regions should be found.

We have studied, in spatial dimension $d=3$, the model with Hamiltonian

$$
-\beta \mathcal{H}=\sum_{\langle i j\rangle}\left[J_{i j} s_{i} s_{j}+K s_{i}^{2} s_{j}^{2}-\Delta\left(s_{i}^{2}+s_{j}^{2}\right)\right],
$$

where $s_{i}=0, \pm 1$ at each site $i$ of the lattice and $\langle i j\rangle$ indicates summation over nearest-neighbor pairs of sites. The spinglass type of quenched randomness is given by each local $J_{i j}$ being ferromagnetic with the value $+J$ with probability 1 $-p$ and antiferromagnetic with the value $-J$ with probability $p$. Under the scale change induced by renormalization-group transformation, all renormalized interactions become quenched random and the more general Hamiltonian

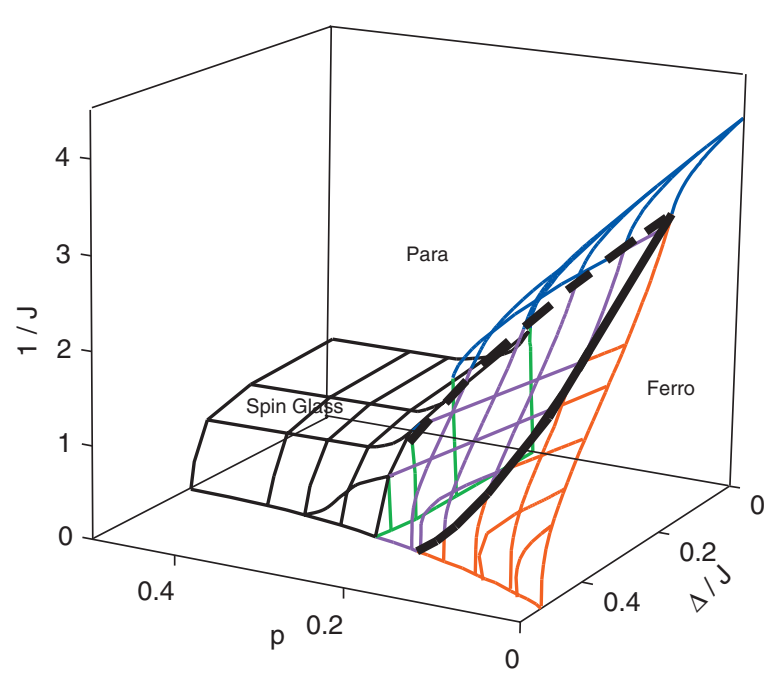

FIG. 1. (Color) Our calculated global phase diagram for $K=0$. The ferromagnetic phase is bounded by a first-order surface (red) close to $p=0$, which recedes along the full line on the surface from a new second-order transition (purple) induced by randomness and controlled by a strong-coupling fixed distribution. At the dashed line, an ordinary second-order transition (blue) takes over. The transitions from the spin-glass phase, to the paramagnetic (black) or ferromagnetic (green) phase, are second order. The system being symmetric about $p=0.5$, the antiferromagnetic sector is not shown. 


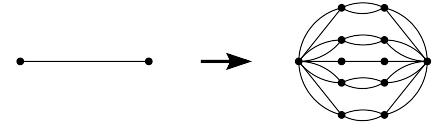

FIG. 2. The $d=3$ hierarchical lattice for which our calculation is exact is constructed by the repeated imbedding of the graph as shown in this figure. This hierarchical lattice gives very accurate results for the critical temperatures of the $d=3$ isotropic and anisotropic Ising models on the cubic lattice [15].

$$
-\beta \mathcal{H}=\sum_{\langle i j\rangle}\left[J_{i j} s_{i} s_{j}+K_{i j} s_{i}^{2} s_{j}^{2}-\Delta_{i j}\left(s_{i}^{2}+s_{j}^{2}\right)-\Delta_{i j}^{\dagger}\left(s_{i}^{2}-s_{j}^{2}\right)\right]
$$

must be considered. The renormalization-group flows are in terms of the joint quenched probability distribution $P\left(J_{i j}, K_{i j}, \Delta_{i j}, \Delta_{i j}^{\dagger}\right)$, which is renormalized through the convolution [14]

$$
P^{\prime}\left(\mathbf{K}_{i^{\prime} j^{\prime}}^{\prime}\right)=\int\left(\prod_{i j}^{i^{\prime} j^{\prime}} d \mathbf{K}_{i j} P\left(\mathbf{K}_{i j}\right)\right) \delta\left(\mathbf{K}_{i^{\prime} j^{\prime}}^{\prime}-\mathbf{R}\left(\left\{\mathbf{K}_{i j}\right\}\right)\right),
$$

where primes refer to the renormalized system, $\mathbf{K}_{i j}$ $\equiv\left(J_{i j}, K_{i j}, \Delta_{i j}, \Delta_{i j}^{\dagger}\right)$, and $\mathbf{R}\left(\mathbf{K}_{i j}\right)$ is the local recursion relation through which 108 unrenormalized local interactions in $\left\{\mathbf{K}_{i j}\right\}$ determine the four renormalized local interactions in $\mathbf{K}_{i^{\prime} j^{\prime}}^{\prime}$. The local recursion relation $\mathbf{R}\left(\mathbf{K}_{i j}\right)$ is effected by a mixed Migdal-Kadanoff procedure [15] with $d=3$ and length rescaling factor $b=3$ necessary for the equal a priori treatment of ferromagnetism and antiferromagnetism. Thus, our treatment is approximate for the cubic lattice and exact for the hierar-

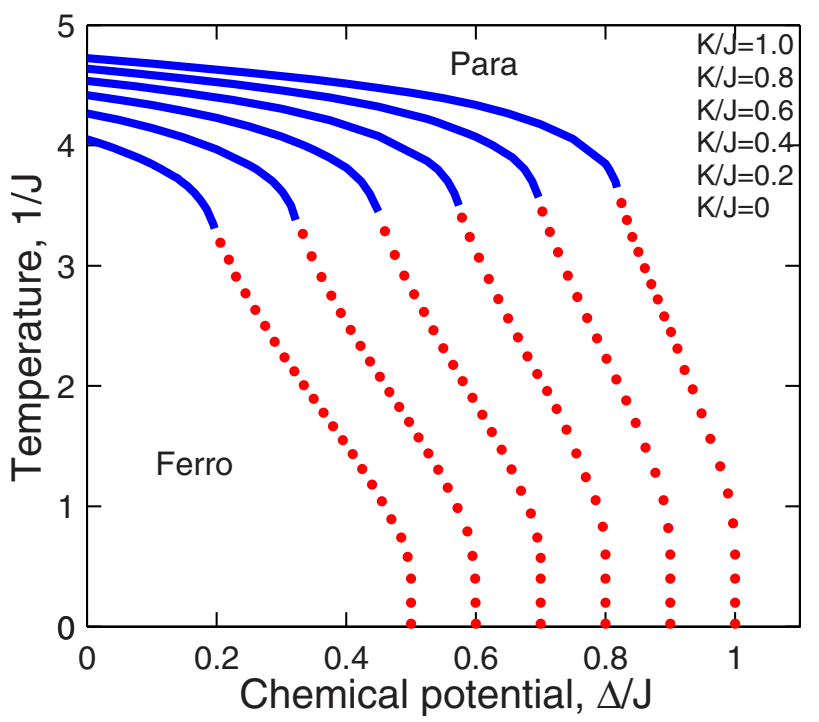

FIG. 3. (Color online) Tricritical phase diagram cross-sections of the purely ferromagnetic system for the $K / J$ values indicated on the figure, shown consecutively from the innermost curve for $K / J$ $=0$. First- and second-order transitions are shown by dotted and full lines, meeting at a tricritical point. In these systems, with no quenched randomness, the standard tricritical topology occurs, with the second-order boundary at high temperature and the first-order boundary at low temperature.

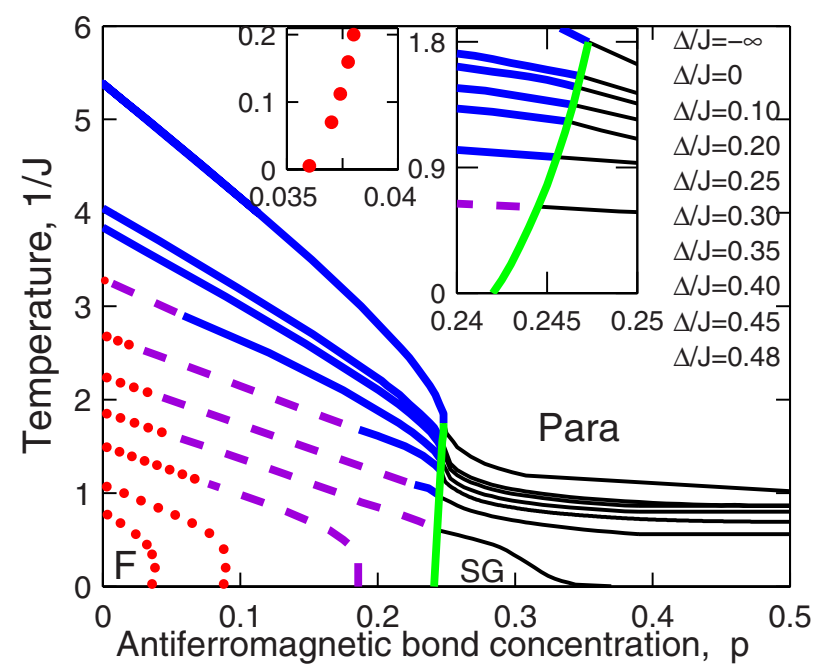

FIG. 4. (Color online) Blume-Emery-Griffiths spin-glass phase diagrams: Constant $\Delta / J$ cross sections of the global phase diagram in Fig. 1. The outermost cross section has $\Delta / J=-\infty$, meaning no $s_{i}=0$ states. The successive cross sections, going inwards from the outermost cross section, are for the successively higher values of $\Delta / J$ indicated on the figure. Thus, annealed vacancies $s_{i}=0$ are introduced in these cross sections with successively higher values of $\Delta / J$, making all ordered phases recede. The dotted and full lines are, respectively, first- and second-order phase boundaries. The dashed lines are strong-coupling second-order phase boundaries induced by quenched randomness. The inverted tricritical topology is seen between the dotted and dashed lines, with the first-order transitions occurring at high temperature and the second-order transitions occurring at low temperature, on each side of the tricritical point. A new spin-glass phase diagram topology is obtained for $\Delta / J=0.35$, in which the spin-glass phase occurs close to the ferromagnetic (and, symmetrically, antiferromagnetic, not shown here) phase, but yields to the paramagnetic phase as $p$ is increased towards 0.5 . The spin-glass phase disappears at $\Delta / J=0.37$. The insets, with expanded scales, clearly show the ferromagnetic to paramagnetic phase reentrance and the ferromagnetic to spin-glass phase reentrance.

chical lattice [15-24] shown in Fig. 2. This hierarchical lattice is known to give very accurate results for the critical temperatures of the $d=3$ isotropic and anisotropic Ising models on the cubic lattice [15]. Furthermore, in general, exact calculations for hierarchical lattices have been seen to constitute very good approximations for cubic lattices [14,24-27].

The probability distribution $P\left(J_{i j}, K_{i j}, \Delta_{i j}, \Delta_{i j}^{\dagger}\right)$ is represented by histograms lodged on a four-dimensional interaction space $\left(J_{i j}, K_{i j}, \Delta_{i j}, \Delta_{i j}^{\dagger}\right)$. Equation (3) is effected by eight pairwise convolutions, which are either bond moving or decimation in the appropriate sequence, between intermediate distributions. The number of histograms rapidly grows from the starting two described after Eq. (1). Thus, for calculational purposes, before each pairwise convolution, the histograms are combined by using a binning procedure, so that our results are obtained by the renormalization-group flows of 22500 histograms.

Tricritical phase diagram cross sections of the purely ferromagnetic system for different $K / J$ values are shown in Fig. 


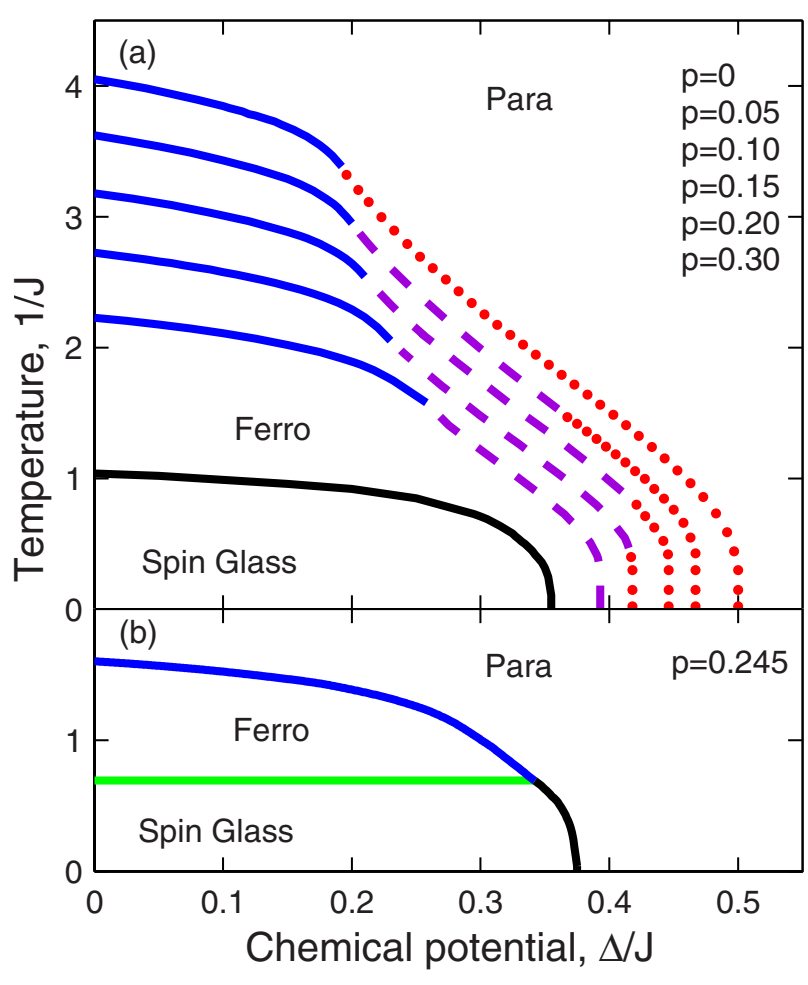

FIG. 5. (Color online) Spin-glass Blume-Emery-Griffiths phase diagrams: Constant $p$ cross sections of the global phase diagram in Fig. 1. The dotted and full lines are, respectively, first- and secondorder phase boundaries. The dashed lines are strong-coupling second-order phase boundaries induced by quenched randomness. The outermost curve in (a) corresponds to the pure Blume-EmeryGriffiths model with no quenched randomness $(p=0)$. The successive cross sections in (a), going inwards from the outermost cross section, are for the successively higher values of $p$ indicated on the figure. As spin-glass quenched randomness is introduced with increasing values of $p$, ordered phases and first-order phase transitions recede.

3. These are standard tricritical phase diagrams, in the absence of quenched randomness, with the tricritical point separating the second-order transitions at high temperature and the first-order transitions at low temperature. The humped boundary, occurring in mean-field theory but not in the $d=2$ system [2], is thus found to occur in the $d=3$ system.

Our calculated global phase diagram for the BEG spinglass system is in Fig. 1 for $K=0$. The ferromagnetic phase is bounded by a first-order surface close to $p=0$, which recedes along the full line on the surface from a new second-order transition induced by randomness and controlled by a strongcoupling fixed distribution. At the dashed line, an ordinary second-order transition takes over. The full line is thus a line of random-bond tricritical points. The dashed line is a line of special critical points around which universality is violated, since the second-order phase transitions on each side of this line have different critical exponents [14]. These two lines meet at the nonrandom $(p=0)$ tricitical point. The transitions from the spin-glass phase, to the paramagnetic or ferromagnetic phase, are second order.

Cross sections of this global phase diagram for constant chemical potential $\Delta / J$ of the non-magnetic state are in Fig. 4. The outermost cross section has $\Delta / J=-\infty$, meaning no $s_{i}$ $=0$ states, and therefore is the phase diagram of the spin- $1 / 2$ Ising spin glass, showing as temperature is lowered the paramagnet-ferromagnet-spin-glass reentrance $[25,26]$. The annealed vacancies, namely the nonmagnetic states $s_{i}=0$, are introduced in cross sections with successively higher values of $\Delta / J$. For $\Delta / J$ greater than the nonrandom tricritical value of $\Delta / J=0.192$, first-order transitions between the ferromagnetic and paramagnetic phases are introduced from the low randomness side, but are converted to the strong-coupling second-order transition at a threshold value of randomness $p$. This constitutes an inverted tricritical point, since the phase boundary is converted from first order to second order as temperature is lowered, contrary to the ordinary tricritical points (as seen, for example, in Fig. 3). The above results are consistent with the general prediction that, in $d=3$, quenched randomness converts first-order boundaries into second order, at a threshold amount of randomness [27]. (In $d=2$, this conversion is predicted to happen with infinitesimal quenched randomness $[27,28]$.) The randomness threshold in $d=3$ is of course higher for stronger first-order transitions. In the current system, increased frustrating quenched randomness has two parallel effects, namely driving the phase transition to lower temperature and reaching the threshold for the conversion to second order, which explains the calculated results of inverted tricriticality. (With nonfrustrating quenched randomness, on the other hand, the transition can actually be driven to higher temperature, while the conversion to second order still happens [29].)

As the annealed vacancies $s_{i}=0$ are increased, at $\Delta / J$ $\geqslant 0.34$, of the second-order transitions between the ferromagnetic and paramagnetic phases, only the strong-coupling transition remains. At $\Delta / J \geqslant 0.42$, the strong-coupling second-order transition also disappears, leaving only firstorder transitions between the ferromagnetic and paramagnetic phases. Also as the annealed vacancies are increased, all ordered phases recede. In this process, first the spin-glass phase disappears, at $\Delta / J=0.37$, which is understandable, since it is tenuously ordered due to frustration. The new, disconnected spin-glass phase diagram topology is obtained in this neighborhood, e.g., for $\Delta / J=0.35$ as shown in Fig. 4, in which the spin-glass phase occurs close to the ferromagnetic (and, symmetrically, antiferromagnetic, not shown in the figures) phase, but yields to the paramagnetic phase as $p$ is increased towards 0.5 . The disconnected spin-glass phase diagrams occur in a very narrow portion of the global phase diagram.

The paramagnetic-ferromagnetic-spin-glass reentrances, as temperature is lowered, of the Blume-Emery-Griffiths spin-glass cross sections fall on the same reentrant secondorder boundary, as seen in Fig. 4. As seen for $\Delta / J=0.45$ and 0.48 in this figure, before disappearing at $\Delta / J=0.5$, the ferromagnetic phase exhibits paramagnetic-ferromagneticparamagnetic reentrance as temperature is lowered.

Constant $p$ cross sections of the global phase diagram in Fig. 1 are shown in Fig. 5. The outermost curve corresponds to the pure Blume-Emery-Griffiths model with no quenched randomness $(p=0)$. As spin-glass quenched randomness is introduced with increasing values of $p$, we see that the first- 
(a)

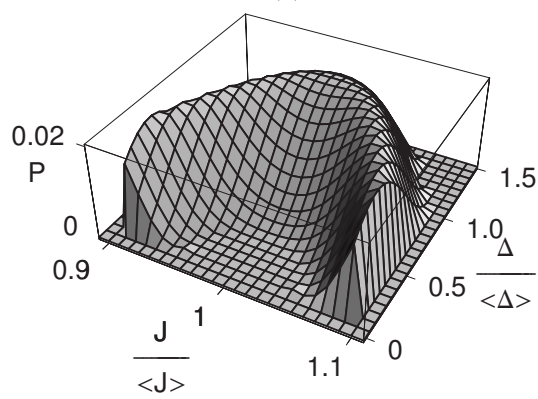

(d)

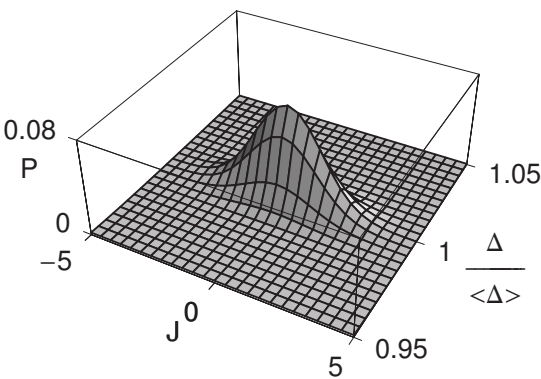

(b)

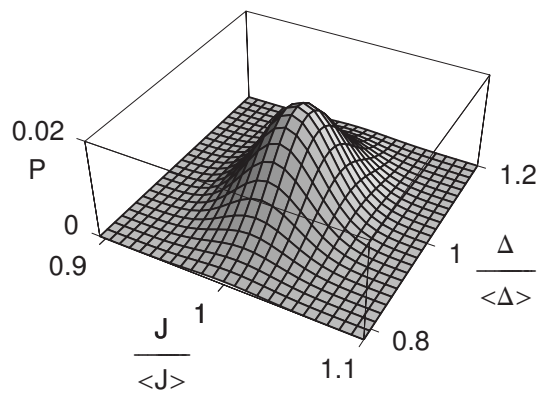

(c)

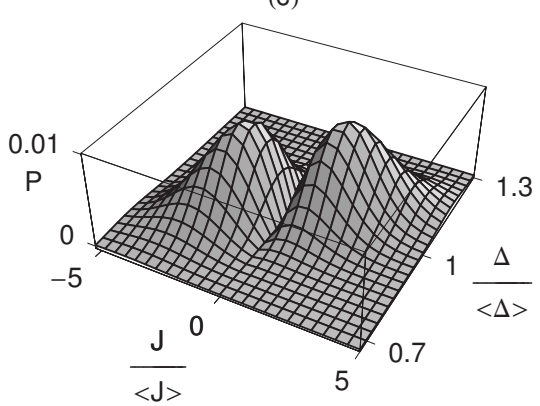

(e)

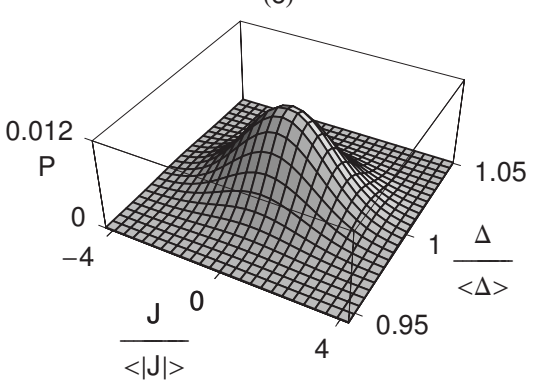

1.05

FIG. 6. Projections of the fixed distributions $P^{*}\left(J_{i j}, K_{i j}, \Delta_{i j}, \Delta_{i j}^{\dagger}\right)$ for (a) the disorder-induced second-order transitions between the ferromagnetic and paramagnetic phases, (b) the first-order transitions between the ferromagnetic and paramagnetic phases, (c) the secondorder transitions between the ferromagnetic and spin-glass phases, (d) the second-order phase transitions between the spin-glass and paramagnetic phases, and (e) the sink fixed distribution for the spin-glass phase. Note that (a),(b),(c),(e) are runaways, in the sense that the couplings renormalize to infinity while the distribution retains its shape shown here. In the second-order phase transitions between the spin-glass and paramagnetic phases (d), $\Delta$ is a runaway (to minus infinity), while the other interactions remain finite. The fixed distributions in this figure are singly unstable, except for the sink (e), which is totally stable.

order boundary recedes to the strong-coupling second-order boundary, while the ordinary second-order boundary also expands. At $p=0.18$, the first-order transition completely disappears. At $p=0.241$, the spin-glass phase appears below the ferromagnetic phase, reflecting complete reentrance. At $p$ $=0.249$, the spin-glass phase completely replaces the ferromagnetic phase as the ordered phase, which is enveloped by second-order transitions only. Thus, for $0.241<p<0.759$, the second-order boundary between the spin-glass and paramagnetic phases reaches zero temperature.

In the results above, the phase diagrams are determined by the basins of attraction of the renormalization-group sinks, namely the completely stable fixed points and fixed distributions: Each basin is a thermodynamic phase. The nature of the phase transitions is determined by analysis of the unstable fixed points and fixed distributions to which the phase diagram points of these transitions flow. Figure 6 shows the unstable fixed distributions of (a) the quenched randomness-induced second-order transitions between the ferromagnetic and paramagnetic phases, (b) the first-order transitions between the ferromagnetic and paramagnetic phases, (c) the second-order transitions between the ferromagnetic and spin-glass phases, and (d) the second-order transitions between the spin-glass and paramagnetic phases. The (totally stable) sink fixed distribution of the spin-glass phase is also shown, in (e). The eigenvalue exponent of the unstable fixed distribution controlling (b) the first-order transitions between the ferromagnetic and paramagnetic phases is $y=3=d$, as is required for first-order transitions [30]. The eigenvalue exponents of the other unstable fixed distributions, (a),(c),(d), are $y<d$ as is required for second-order transitions.

We thank C. Güven M. Hinczewski, C. N. Kaplan, and O. S. Sarıyer for comments. This research was supported by the Scientific and Technological Research Council (TÜBİTAK) and by the Academy of Sciences of Turkey.
[1] M. Blume, V. J. Emery, and R. B. Griffiths, Phys. Rev. A 4, 1071 (1971).

[2] A. N. Berker and M. Wortis, Phys. Rev. B 14, 4946 (1976).

[3] W. Hoston and A. N. Berker, Phys. Rev. Lett. 67, 1027 (1991).

[4] J. Lajzerowicz and J. Sivardière, Phys. Rev. A 11, 2079 (1975).

[5] J. Sivardière and J. Lajzerowicz, Phys. Rev. A 11, 2090 (1975); 11, 2101 (1975).
[6] M. Schick and W.-H. Shih, Phys. Rev. B 34, 1797 (1986).

[7] K. E. Newman and J. D. Dow, Phys. Rev. B 27, 7495 (1983).

[8] T. Tanaka and T. Kawabe, J. Phys. Soc. Jpn. 54, 2194 (1985).

[9] S. A. Kivelson, V. J. Emery, and H. Q. Lin, Phys. Rev. B 42, 6523 (1990).

[10] H. Nishimori, Statistical Physics of Spin Glasses and Information Processing (Oxford University Press, Oxford, 2001).

[11] J. J. Arenzon, M. Nicodemi, and M. Sellitto, J. Phys. I 6, 1143 
(1996).

[12] M. Sellitto, M. Nicodemi, and J. J. Arenzon, J. Phys. I 7, 945 (1997).

[13] J. J. Arenzon, F. Ricci-Tersenghi, and D. A. Stariolo, Phys. Rev. E 62, 5978 (2000).

[14] A. Falicov and A. N. Berker, Phys. Rev. Lett. 76, 4380 (1996).

[15] A. Erbaş, A. Tuncer, B. Yücesoy, and A. N. Berker, Phys. Rev. E 72, 026129 (2005).

[16] A. N. Berker and S. Ostlund, J. Phys. C 12, 4961 (1979).

[17] R. B. Griffiths and M. Kaufman, Phys. Rev. B 26, 5022 (1982).

[18] M. Kaufman and R. B. Griffiths, Phys. Rev. B 30, 244 (1984).

[19] M. Hinczewski and A. N. Berker, Phys. Rev. E 73, 066126 (2006).

[20] M. Hinczewski, Phys. Rev. E 75, 061104 (2007).

[21] Z. Zhang, L. Rong, and S. Zhou, Physica A 377, 329 (2007).
[22] H. D. Rozenfeld, S. Havlin, and D. ben-Avraham, New J. Phys. 9, 175 (2007).

[23] E. Khajeh, S. N. Dorogovtsev, and J. F. F. Mendes, Phys. Rev. E 75, 041112 (2007).

[24] C. Monthus and T. Garel, Phys. Rev. B 77, 134416 (2008).

[25] G. Migliorini and A. N. Berker, Phys. Rev. B 57, 426 (1998).

[26] F. D. Nobre, Phys. Rev. E 64, 046108 (2001).

[27] K. Hui and A. N. Berker, Phys. Rev. Lett. 62, 2507 (1989); 63, 2433(E) (1989).

[28] M. Aizenman and J. Wehr, Phys. Rev. Lett. 62, 2503 (1989); 64, 1311(E) (1990).

[29] A. Malakis, A. N. Berker, I. A. Hadjiagapiou, and N. G. Fytas (unpublished).

[30] B. Nienhuis and M. Nauenberg, Phys. Rev. Lett. 35, 477 (1975). 